\title{
Correlation Free Forms for Nonlinear Stochastic Systems*
}

\author{
S. M. MeERKov AND T. RunOLFSSON \\ Department of Electrical Engineering and Computer Science, \\ The University of Michigan, Ann Arbor, Michigan 48109-2122
}

\author{
AND \\ Z. ScHUSS \\ School of Mathematical Sciences, Tel-Aviv University, \\ Tel-Aviv, Israel
}

Submitted by S. M. Meerkov

Received November 18, 1987

\begin{abstract}
An asymptotic method for analysis of nonlinear systems with wide-band and high frequency stochastic perturbation is developed. Correlation free (Itô) limiting equations are derived and a generalization of the Wong-Zakai correction for the drift term is obtained. A correction for the diffusion term is also shown to exist. Several illustrative examples are considered. 1989 Academic Press, Inc.
\end{abstract}

\section{INTRODUCTION}

Dynamical systems with stochastic perturbations are of significant importance in a number of applications. Direct analyses of such systems are usually prohibitively difficult. A possibility of simplification is offered by derivation of a correlation free form for a system under consideration: such a form admits, at the least, an elementary analysis of deterministic counterpart of the stochastic system. Itô form for a stochastic system given in the Stratonovich description is an example of such a simplification. However, the Itô-Stratonovich connection (i.e., the Wong-Zakai formula [1]) is derived only for dynamical systems with wide-band noises. Although such noises are indeed of prime importance, other applications may involve high frequency perturbation as well. It is the goal of this paper

* This work was supported by the U.S. Department of Energy under Grant DE-FG0285ER 13315. 
to derive a correlation free form for dynamical systems with both wideband and high frequency perturbations and, in this manner, to generalize the Wong-Zakai theory.

More specifically, we study here systems of the form

$$
\begin{gathered}
\frac{d x}{d t}=X_{0}(x)+\frac{1}{\sqrt{\varepsilon}} X_{1}\left(x, \xi_{1}(t / \varepsilon)\right)+\frac{1}{\varepsilon} X_{2}\left(x, \xi_{2}(t / \varepsilon)\right), \\
x \in \mathbb{R}^{n}, X_{0}: \mathbb{R}^{n} \rightarrow \mathbb{R}^{n}, \xi_{i} \in \mathbb{R}^{m_{i}}, X_{i}: \mathbb{R}^{n} \times \mathbb{R}^{m_{i}} \rightarrow \mathbb{R}^{n}, i=1,2, \\
0<\varepsilon \ll 1,
\end{gathered}
$$

where $X_{0}(x)$ represents the deterministic unperturbed dynamics while $(1 / \sqrt{\varepsilon}) X_{1}\left(x, \xi_{1}(t / \varepsilon)\right)$ and $(1 / \varepsilon) X_{2}\left(x, \xi_{2}(t / \varepsilon)\right)$ are intended to model wideband and high frequency (state dependent) perturbations, respectively. To accomplish the latter, we assume that for each fixed $x \in \mathbb{R}^{n}$, $(1 / \sqrt{\varepsilon}) X_{1}\left(x, \xi_{1}(t / \varepsilon)\right)$ is a vector, stationary, ergodic, wide-band random process and $(1 / \varepsilon) X_{2}\left(x, \xi_{2}(t / \varepsilon)\right)$ is either a vector, stationary, ergodic high frequency random process, i.e., a process whose power spectral density vanishes, at least quadratically, at 0 , or an almost periodic vector function. To avoid trivialities, it is also assumed that

$$
\begin{aligned}
& E\left[X_{1}\left(x, \xi_{1}(\tau)\right) \mid x\right] \equiv 0, \\
& E\left[X_{2}\left(x, \xi_{2}(\tau)\right) \mid x\right] \equiv 0,
\end{aligned}
$$

where $E$ denotes averaging. Although (1.2) holds for each fixed $x$, there may be nonzero correlation between state $x(t)$ and processes $\xi_{i}(\tau), i=1,2$, in Eq. (1.1). This would imply in particular that even if $X_{0}(x)=A x$,

$$
\frac{d E x}{d t} \neq X_{0}(E x)
$$

and therefore, the averaging would not reveal the deterministic counterpart of the stochastic system.

In this paper, we derive asymptotic (with respect to $\varepsilon \rightarrow 0$ ) correlation free forms of (1.1), (1.2), i.e., limiting equations in which the noises and the states are uncorrelated. An analogous problem has been addressed in $[2,3]$ for situations in which $X_{2}\left(x, \xi_{2}\right) \equiv 0$, and in $[4,5]$ for situations with $X_{1}\left(x, \xi_{1}\right) \equiv 0$, and $\xi_{2}$ an almost periodic function. Results for the general case are derived below.

Specifically, we show that the interaction of $\xi_{1}$ and $\xi_{2}$ brings about not only a generalization of the Wong-Zakai formula for the drift coefficient in the limiting Itô equation but also a correction in the diffusion term. The latter implies that the limiting equation may contain more independent 
white noises than wide-band noises of the original system (1.1). This phenomenon has its origin in the modulation of $\xi_{1}$ by $\xi_{2}$ which, in the limit of $\varepsilon \rightarrow 0$, results in a new, independent white noise.

The results obtained here may find applications for stability analysis of systems with random perturbations and, in particular, for derivation of vibrational stabilizability conditions for stochastic systems $[5,6]$.

The structure of the paper is as follows: In Section 2 we derive correlation free forms for Fq. (1.1) and analyze their properties on finite and infinite time intervals; in Section 3 several examples are considered; the formal derivations are given in the Appendix. The development is based on a combination of two techniques, the first of which has its origin in [4] and the second in [3].

\section{Main Results}

In order to reduce Eq. (1.1) to a form suitable for asymptotic analysis, introduce the generating equation [4] of the form

$$
\frac{d x}{d \tau}=X_{2}\left(x, \xi_{2}(\tau)\right), \quad \tau=t / \varepsilon
$$

Assume that (2.1) has a unique solution

$$
x(\tau)=h\left(\tau, x_{0}\right)
$$

defined for every initial condition $x_{0} \in \mathbb{R}^{n}$ for all $\tau \geqslant 0$.

The following proposition was proved in [4].

Proposition 2.1. Assume that $X_{2}\left(x, \xi_{2}\right)$ is differentiable with respect to $x$. In this case substitution

$$
x(\tau)=h(\tau, y(\tau))
$$

reduces (1.1) to the standard form

$$
\frac{d y}{d t}=Y_{0}(y, t / c)+\frac{1}{\sqrt{\varepsilon}} Y_{1}\left(y, t / c, \xi_{1}(t / \varepsilon)\right)
$$

where

$$
Y_{i}\left(y, t / \varepsilon, \xi_{i}(t / \varepsilon)\right)=\left[\frac{\partial h}{\partial y}(t / \varepsilon, y)\right]^{-1} X_{i}\left(h(t / \varepsilon, y), \xi_{i}(t / \varepsilon)\right), \quad i=0,1, \quad \xi_{0} \equiv 0 .
$$


Under appropriate assumptions on functions $X_{i}$ and the noise processes $\xi_{i}(\tau)$, the asymptotic methods of $[2,3]$ can be utilized to analyze the asymptotic behaviour of $(2.4)$ as $\varepsilon \rightarrow 0$. Specifically, assume that $X_{i}$, $i=0,1,2$, are smooth functions of $x$, continuous in $\xi_{i}$ and that $X_{0}, X_{1}$, and $h$ satisfy the following conditions.

There exists a constant $C>0$, independent of $\tau$ and $\xi_{1}(\tau)$, such that for all $x \in \mathbb{R}^{n}$

(i) $\left|X_{1}\left(x, \xi_{1}\right)\right| \leqslant C(1+|x|),|h(x, \tau)| \leqslant C(1+|x|)$.

(ii) $\left|\partial X_{0} / \partial x\right| \leqslant C,\left|\left(\partial X_{i} / \partial x\right)\left(x, \xi_{i}\right)\right| \leqslant C,|(\partial h / \partial x)(x, \tau)| \leqslant C$.

(iii) Higher order $x$-derivatives of $h$ and $X_{i}, i=0,1$, are bounded by powers of $|x|$ uniformly in $\tau$ and $\xi_{1}$.

We make the following assumptions about $\xi_{1}(\tau)$, and $h\left(\tau, x_{0}\right)$ :

(a) $\xi_{1}(\tau)$ is independent of $\xi_{2}(\tau)$.

(b) $\xi_{1}(\tau)$ is an ergodic, stationary diffusion process with a transition function $P\left(t, \xi_{1}, A\right)$ and unique invariant probability measure $\mu(A)$, such that

$$
\mu(A)=\lim _{t \rightarrow \infty} P\left(t, \xi_{1}, A\right)
$$

uniformly in $\xi_{1}$ and $A \subset \mathbb{R}^{m_{1}}$. Furthermore, the recurrent potential kernel

$$
Q\left(\xi_{1}, A\right)=\int_{0}^{\infty}\left(P\left(t, \xi_{1}, A\right)-\mu(A)\right) d t
$$

exists and maps the bounded smooth functions of $\xi_{1}$ into themselves [3].

(c) $h\left(\tau, x_{0}\right)$ satisfies the ergodicity condition

$$
\lim _{T \rightarrow \infty} \frac{1}{T} \int_{0}^{T} h\left(\tau, x_{0}\right) d t=h\left(x_{0}\right), \quad \forall x_{0} \in \mathbb{R}^{n},
$$

where $h: \mathbb{R}^{n} \rightarrow \mathbb{R}^{n}$ is a deterministic function.

Let $Y_{i}^{j}$ be the $j$ th component of $Y_{i}, i=0,1, j=1, \ldots, n$. Define

$$
\begin{aligned}
\bar{Y}_{0}^{\prime}(y)= & \lim _{T \rightarrow \infty} \frac{1}{T} \int_{t_{0}}^{T} E\left[Y_{0}^{j}(y, \tau)\right] d \tau \\
& +\lim _{T \rightarrow \infty} \frac{1}{T} \int_{t_{0}}^{T} \int_{t_{0}}^{\tau} \sum_{i=1}^{n} E\left[Y_{1}^{i}\left(y, \sigma, \xi_{1}(\sigma)\right) \frac{\partial Y_{1}^{j}\left(y, \tau, \xi_{1}(\tau)\right)}{\partial y_{i}}\right] d \sigma d \tau,(2.6) \\
a_{i j}(y)= & \lim _{T \rightarrow \infty} \frac{1}{T} \int_{t_{0}}^{T} \int_{t_{0}}^{\tau} E\left[Y_{1}^{j}\left(y, \tau, \xi_{1}(\tau)\right) Y_{1}^{i}\left(y, \sigma, \xi_{1}(\sigma)\right)\right] d \sigma d \tau
\end{aligned}
$$


and

$$
\bar{L}=\sum_{j=1}^{n} \bar{Y}_{0}^{j}(y) \frac{\partial}{\partial y_{j}}+\sum_{i, j=1}^{n} a_{i j}(y) \frac{\partial^{2}}{\partial y_{i} \partial y_{j}} .
$$

The following limit theorem can be proved using the techniques of [3].

THEOREM 2.1. Assume that in addition to the aforementioned assumptions we have

(i) the limits (2.6) exist uniformly in $y$ and are independent of $t_{0}$;

(ii) $\lim _{T \rightarrow \infty}(1 / T) \int_{0}^{T} E\left[Y_{1}\left(y, \tau, \xi_{1}\right)\right] d \tau=0$.

Then $y(t), t \geqslant 0$, defined by (2.4) converges weakly in $C\left([0, T] ; \mathbb{R}^{n}\right), T<\infty$, as $\varepsilon \rightarrow 0$ to the time-homogeneous diffusion $\bar{y}(t)$ generated by $L$.

Remark 2.1. The diffusion process $\bar{y}(t)$ generated by $\bar{L}$ can also be represented as the solution of the Itô equation

$$
d \bar{y}=\bar{Y}_{0}(\vec{y}) d t+\bar{Y}_{1}(\bar{y}) d w
$$

where $w(t)$ is a standard $n$-dimensional Brownian motion and $\left(\bar{Y}_{1}(y) \bar{Y}_{1}^{T}(y)\right)_{i j}=2 a_{i j}(y)$. Equation $(2.8)$ is a correlation free form of system (2.4); i.e., the noise $d w(t)$ and the state $\bar{y}(t)$ are uncorrelated. The second term of $\bar{Y}_{0}(\bar{y})$ in (2.6) is the generalized Wong-Zakai correction for the drift, and a correction due to the high frequency process $\xi_{2}(\tau)$ is present in the diffusion term $\bar{Y}_{1}(\bar{y})$.

The following stability theorem can also be proved using the techniques of [3].

THEOREM 2.2. Assume that in addition to the assumptions of Theorem 2.1 we have

$$
Y_{i}=0 \quad \text { if } \quad y=0, i=0,1
$$

i.e., 0 is an equilibrium point of (2.4). Assume there exists a smooth positive definite function $V(\bar{y})$ on $\mathbb{R}^{n}$ such that

$$
\bar{L} V(\bar{y}) \leqslant-\gamma V(\bar{y}), \quad \gamma>0,
$$

for all $\bar{y}$ belonging to some open neighborhood of 0 . Then there exists an $\varepsilon_{0}>0$ such that for all $0<\varepsilon \leqslant \varepsilon_{0}, 0$ is a uniformly stochastically exponentially stable equilibrium point of $(2.4)$; i.e., for all $\eta_{1}, \eta_{2}>0$ there exists $\delta>0$ such that if $|y(0)|<\delta$ then

$$
P\left\{|y(t)| \leqslant \eta_{2} e^{-\tilde{\gamma} t}, t \geqslant 0\right\} \geqslant 1-\eta_{1}, \quad \tilde{\gamma}>0 .
$$


Remark 2.2. Other conditions for convergence on infinite time-intervals exist. In particular, if $\bar{y}(t)$ is ergodic it follows from the results of [7] that $y(t)$ converges to $\bar{y}(t)$ in distribution on $(0, \infty)$.

The proofs of Theorems 2.1 and 2.2 are almost identical to the proofs of Theorems 4 and 5.1 in [3] and will be omitted here. Instead we outline the derivation of (2.6), (2.7) in the Appendix.

Theorems 2.1 and 2.2 establish the relationship between the process $y(t)$ and its limiting process $\bar{y}(t)$ on finite and infinite time intervals. On the other hand, process $x(t)$ defined by (1.1) is related to $y(t)$ through substitution (2.3). Define process $\bar{x}(t)$ by

$$
\bar{x}(t)=h(\tau, \bar{y}(t))
$$

where $\bar{y}(t)$ is given by (2.8). Then we have the following proposition.

Proposition 2.2. Assume that the hypotheses of Theorem 2.1 are true. Then

(i) $x(t)$ defined by (1.1) converges weakly in $C\left([0, T] ; \mathbb{R}^{n}\right)$ as $\varepsilon \rightarrow 0$ to $\bar{x}(t)$ defined by $(2.11)$.

(ii) If (2.9) and (2.10) hold, then there exists an $\varepsilon_{0}>0$ such that for all $0<\varepsilon \leqslant \varepsilon_{0}$ and $\hat{\eta}_{1}, \hat{\eta}_{2}>0$ there exists a $\delta>0$ such that if $x(0)=\bar{x}(0)$ and $|x(0)|<\delta$ then

$$
P\left\{\sup _{t \geqslant 0}|x(t)-\bar{x}(t)| \leqslant \hat{\eta}_{2}\right\} \geqslant 1-\hat{\eta}_{1} .
$$

Proof. See the Appendix.

Remark 2.3. The differential equation for $\bar{x}(t)$ defined in $(2.11)$ is

$$
d \bar{x}=\bar{X}_{0}(\tau, \bar{x}) d t+\bar{X}_{1}(\tau, x) d w+\frac{1}{\varepsilon} X_{2}\left(\bar{x}, \xi_{2}(\tau)\right) d t,
$$

where

$$
\bar{X}_{i}(\tau, \bar{x})=\frac{\partial h}{\partial y}(\tau, g(\tau, \bar{x})) \bar{Y}_{i}(g(\tau, \bar{x})), \quad i=0,1
$$

and $g(\tau, \bar{x})$ is the inverse of $h(\tau, \bar{y})$ in the $\bar{y}$ variables, i.e., $\bar{x}=h(\tau, g(\tau, \bar{x}))$. It is easy to show that

$$
\lim _{T \rightarrow \infty} \frac{1}{T} \int_{0}^{T} X_{2}\left(\bar{x}(\tau, t), \xi_{2}(\tau)\right) d \tau \equiv 0 .
$$


Since, $E\left[\bar{X}_{1}(\tau, \bar{x}) d w\right]=0$, Eq. (2.12) can be viewed as a correlation free form of (1.1) and the difference between $\bar{X}_{0}$ and $\bar{X}_{1}$ and their counterparts in (1.1) can be viewed as generalized Wong-Zakai corrections in $x$-space. However, since $\bar{x}$ and $\bar{y}$ have a simple relationship (2.11), Eq. (2.12) does not offer additional information or utility in comparison with the correlation free form $(2.8)$.

\section{EXAMPLES}

Below we present three examples. The first one is the linear harmonic oscillator with random spring constant $[3,8]$. We assume that the random process is a sum of independent wide-band and high frequence processes and analyze the effect of each process on the stability properties of the oscillator. The second example is a simple linear system in which the generalized Wong-Zakai correction in the diffusion term is clearly illustrated. The last example is the Rayleigh oscillator with wide-band parametric oscillations and high frequency forcing. In this example the stabilizing effect of the high frequency noise is illustrated.

EXAMPLE 1. Consider the linear harmonic oscillator with damping coefficient $\gamma$ and a random spring constant

$$
\ddot{x}+2 \gamma \dot{x}+\left(\omega^{2}+\xi(t)\right) x=0,
$$

where

$$
\xi(t)=\frac{1}{\sqrt{\varepsilon}} F\left(\xi_{1}(t / \varepsilon)\right)+\frac{1}{\varepsilon} \xi_{2}(t / \varepsilon),
$$

$\xi_{1}(\tau)$ is a one-dimensional Ornstein-Uhlenbeck process

$$
d \xi_{1}=-\xi_{1} d \tau+d w
$$

and $F: \mathbb{R} \rightarrow I=(a, b)$ (a bounded interval) is such that

$$
E\left\{F\left(\xi_{1}\right)\right\}=0 ;
$$

$\xi_{2}(\tau)$ is any integrable, stationary, ergodic, high frequency process whose integral $\eta(\tau)=\int \xi_{2}(\tau) d \tau$ is also stationary and ergodic and bounded.

The generating equation for $(3.1)$ is

$$
\begin{aligned}
& \dot{x}_{1}=0 \\
& \dot{x}_{2}=\frac{1}{\varepsilon} \xi_{2}(t / \varepsilon) x_{1}
\end{aligned}
$$


and the substitution (2.3) becomes $x_{1}=y_{1}, x_{2}=\eta(\tau) y_{1}+y_{2}$. The equation in the standard form is

$\dot{y}_{1}=y_{2}+\eta(t / \varepsilon) y_{1}$

$\dot{y}_{2}=-(2 \gamma+\eta(t / \varepsilon)) y_{2}-\left(\omega^{2}+\eta^{2}(t / \varepsilon)-2 \gamma \eta(t / \varepsilon)+\frac{1}{\sqrt{\varepsilon}} F\left(\xi_{1}(t / \varepsilon)\right)\right) y_{1}$.

The averaged equation corresponding to (3.3) is

$$
\begin{aligned}
& \dot{\bar{y}}_{1}=\bar{y}_{2} \\
& \dot{\bar{y}}_{2}=-2 \gamma \bar{y}_{2}-\left(\omega^{2}+\alpha^{2}\right) \bar{y}_{1}+\sigma \bar{y}_{1} \dot{w},
\end{aligned}
$$

where $\dot{w}$ is a standard Gaussian white noise, $\alpha^{2}$ is the variance of $\eta(\tau)$,

$$
\alpha^{2}=\lim _{T \rightarrow \infty} \frac{1}{T} \int_{0}^{T} \eta^{2}(\tau) d \tau
$$

and

$$
\begin{aligned}
\sigma^{2} & \left.=\int_{-\infty}^{\infty} E\left\{F \xi_{2}(s)\right) F\left(\xi_{2}(0)\right)\right\} d s \\
& =\int_{-\infty}^{\infty} R(s) d s .
\end{aligned}
$$

The differential generator of the averaged diffusion process (3.4) is

$$
\bar{L}=\bar{y}_{2} \frac{\partial}{\partial \bar{y}_{1}}-\left(2 \gamma \bar{y}_{2}+\left(\omega^{2}+\alpha^{2}\right) \bar{y}_{1}\right) \frac{\partial}{\partial \bar{y}_{2}}+\frac{\sigma^{2}}{2} \bar{y}_{1}^{2} \frac{\partial^{2}}{\partial \bar{y}_{2}} .
$$

To analyze the stability properties of (3.4) and, therefore, of (3.3) and (3.1) for sufficiently small $\varepsilon$ we solve the equation

$$
\bar{L} V(\bar{y})=-\left(c_{1} \bar{y}_{1}^{2}+c_{2} \bar{y}_{2}^{2}\right), \quad c_{i}>0
$$

with

$$
V(\bar{y})=m_{11} \bar{y}_{1}^{2}+2 m_{12} \bar{y}_{1} \bar{y}_{2}+m_{22} \bar{y}_{2}^{2} .
$$

A simple calculation shows that the quadratic form (3.7) is positive definite for all $c_{i}>0, i=1,2$, if and only if

$$
2\left(\omega^{2}+\alpha^{2}\right) \gamma>\frac{\sigma^{2}}{2}
$$


Conditions of the form (3.8) have been derived from [3, 8]. However, there is an interesting difference here. Indeed, rewriting (3.8) as

$$
4 \gamma>\frac{\sigma^{2}}{\omega^{2}+\alpha^{2}}
$$

we note that strong wide-band noise (large $\sigma^{2}$ ) requires a large damping coefficient $\gamma$, whereas large high frequency noise (large $\alpha^{2}$ ) reduces the bound (3.9) on $\gamma$. Thus, since (3.9) is a necessary condition for stability of (3.1) we conclude that the wide-band noise destabilizes (3.1) while the high frequency noise is stabilizing.

EXAmple 2. Consider the linear system

$$
\begin{aligned}
& \dot{x}_{1}=-x_{1}+\frac{1}{\sqrt{\varepsilon}} F\left(\xi_{1}(t / \varepsilon)\right) \\
& \dot{x}_{2}=-2 x_{2}+\frac{1}{\varepsilon}(\sin t / \varepsilon) x_{1},
\end{aligned}
$$

where $F\left(\xi_{1}(t / \varepsilon)\right)$ is a wide-band process as in Example 1. The generating equation for $(3.10)$ is

$$
\begin{aligned}
& \dot{x}_{1}=0 \\
& \dot{x}_{2}=\frac{1}{\varepsilon}(\sin t / \varepsilon) x_{1},
\end{aligned}
$$

and the substitution (2.3) is $x_{1}=y_{1}, x_{2}=-(\cos \tau) y_{1}+y_{2}$. The system in standard form is

$$
\begin{aligned}
& \dot{y}_{1}=-y_{1}+\frac{1}{\sqrt{\varepsilon}} F\left(\xi_{2}(t / \varepsilon)\right) \\
& \dot{y}_{2}=-2 y_{2}+(\cos t / \varepsilon) y_{1}+\frac{1}{\sqrt{\varepsilon}} F\left(\xi_{2}(t / \varepsilon)\right) \cos t / \varepsilon
\end{aligned}
$$

and the averaged system

$$
\begin{aligned}
& \dot{\bar{y}}_{1}=-\bar{y}_{1}+\sigma \dot{w}_{1} \\
& \dot{\bar{y}}_{2}=-2 \bar{y}_{2}+\frac{\sigma}{\sqrt{2}} \dot{w}_{2},
\end{aligned}
$$

where $\dot{w}_{1}$ and $\dot{w}_{2}$ are independent white noise processes. The noise process $\dot{w}_{2}$ is obtained as the limit $\varepsilon \rightarrow 0$ of the modulated wide-band proces $(1 / \sqrt{\varepsilon}) F\left(\xi_{1}(t / \varepsilon)\right) \cos t / \varepsilon$. Obviously, $(\sigma / \sqrt{2}) \dot{w}_{2}$ is the generalized diffusion correction. 
The limiting system (3.13) is a pair of ergodic, independent, Ornstein-Uhlenbeck processes. Hence, it has a unique stationary distribution and it follows from [7] that $y(t)$ and $\bar{y}(t)$ are close in distribution for small $\varepsilon$ and all $t \geqslant 0$. Furthermore, it follows that the solution of (3.10) is close in distribution to $\bar{x}=\left[\bar{y}_{1},-(\cos (t / \varepsilon)) \bar{y}_{1}+\bar{y}_{2}\right]$ for all $t \geqslant 0$ and small $\varepsilon>0$.

EXAMPLE 3. Consider the Rayleigh equation with parametric oscillations and forcing

$$
\ddot{x}+\mu\left(\dot{x}^{3} / 3-\dot{x}\right)+\left(1+\frac{1}{\sqrt{\varepsilon}} F\left(\xi_{1}(t / \varepsilon)\right)\right) x=\frac{1}{\varepsilon} \xi_{2}(t / \varepsilon)
$$

or equivalently the system

$$
\begin{aligned}
& \dot{x}_{1}=x_{2} \\
& \dot{x}_{2}=-\mu\left(x_{2}^{3} / 3-x_{2}\right)-\left(1+\frac{1}{\sqrt{\varepsilon}} F\left(\xi_{1}(t / \varepsilon)\right)\right) x_{1}+\frac{1}{\varepsilon} \xi_{2}(t / \varepsilon) .
\end{aligned}
$$

We assume that $F\left(\xi_{1}\right)$ and $\xi_{2}$ satisfy the assumptions of Example 1. The change of coordinates for (3.15) is $x_{1}=y_{1}, x_{2}=y_{2}+\eta$, and the equation in standard form

$\dot{y}_{1}=y_{2}+\eta(t / \varepsilon)$

$\dot{y}_{2}=-\mu\left(\left(y_{2}+\eta(t / \varepsilon)\right)^{3} / 3-\left(y_{2}+\eta(t / \varepsilon)\right)\right)-\left(1+\frac{1}{\sqrt{\varepsilon}} F\left(\xi_{1}(t / \varepsilon)\right)\right) y_{1}$.

The average equation corresponding to (3.16) is

$$
\begin{aligned}
& \dot{\bar{y}}_{1}=\bar{y}_{2} \\
& \dot{\bar{y}}_{2}=-\mu\left(y_{2}^{3} / 3+\left(\alpha^{2}-1\right) \bar{y}_{2}\right)-\bar{y}_{1}+\sigma \bar{y}_{1} \dot{w} .
\end{aligned}
$$

Systems (3.16) and (3.17) have equilibrium point at $(0,0)$. To investigate the stability properties of (3.16) in a neighborhood of zero we linearize $(3.17)$ around $(0,0)$. The resulting linear system is

$$
\begin{aligned}
& \dot{z}_{1}=z_{2} \\
& \dot{z}_{2}=-\mu\left(\alpha^{2}-1\right) z_{2}-z_{1}+\sigma z_{1} \dot{w} .
\end{aligned}
$$

A simple calculation shows that (3.18) is uniformly, stochastically, exponentially stable if

$$
\alpha^{2}>1+\frac{\sigma^{2}}{2 \mu}
$$


Therefore, by [9] and Theorem 2.2, (3.17) and thus (3.16) (for small enough $\varepsilon$ ) are also asymptotically stable in a neighborhood of zero if (3.19) is satisfied. Finally, since $x_{1}=y_{1}$ and $x_{2}=y_{2}+\eta(t / \varepsilon)$, we conclude that if (3.19) is satisfied then (3.15) has an asymptotically stable ergodic solution $\left(x_{1}^{s}(t), x_{2}^{s}(t)\right)$. Thus, the high frequency oscillations in (3.14) have resulted in a transition of the unstable equilibrium point $(0,0)$ of the system

$$
\ddot{x}+\mu\left(\dot{x}^{3} / 3-\dot{x}\right)+\left(1+\frac{1}{\sqrt{\varepsilon}} F\left(\xi_{1}(t / \varepsilon)\right)\right) x=0
$$

into an asymptotically stable ergodic solution.

\section{APPENDIX}

First we outline the derivation of (2.6), (2.7). Define for smooth functions $f: \mathbb{R}^{n} \rightarrow \mathbb{R}$

$$
Q_{f}\left(y, \tau, \xi_{1}\right)=\int_{0}^{\infty} d s \int_{\mathbb{R}^{m_{1}}}\left(P\left(s, \xi_{1}, d z\right)-\mu(d z)\right) \sum_{j=1}^{n} Y_{1}^{j}(y, \tau+s, z) \frac{\partial f}{\partial y_{j}} .
$$

Condition the process $y(t)$, given by $(2.4)$, on a trajectory of $h(\tau, \cdot)$, i.e., let $\mathbf{H}_{\tau}$ be the $\sigma$-algebra generated by $h(s, \cdot), 0 \leqslant s \leqslant \tau$. Then since $\xi_{1}(\tau)$ is independent of $\mathbf{H}_{\tau}$ the joint process $\left(y(t), \xi_{1}(\tau)\right)$ conditioned on $\mathbf{H}_{\tau}$ is a diffusion process on $\mathbb{R}^{n} \times \mathbb{R}^{m_{1}}$. Its conditional transitional probability density function $p\left(t, y, \xi_{1} \mid \mathbf{H}_{\tau}\right)$ satisfies the backward Kolmogorov equation. We assume that $p$ is a function of slow and fast time $t$ and $\tau=t / \varepsilon$ and write the backward equation in the form

$$
L p=\frac{\partial p}{\partial t}+\frac{1}{\varepsilon} \frac{\partial p}{\partial \tau}+\sum_{i=1}^{n}\left(Y_{0}^{i}+\frac{1}{\sqrt{\varepsilon}} Y_{1}^{i}\right) \frac{\partial p}{\partial y_{i}}+\frac{1}{\varepsilon} K p=0,
$$

where $K$ is the differential generator of the process $\xi_{1}(\tau)$. Next we derive an averaged equation for $y$ by expanding $p\left(y, t, \tau, \xi_{1} \mid \mathbf{H}_{\tau}\right)$ in power series in $\sqrt{\varepsilon}$. Rewriting (A.1) in the obvious operator notation

$$
L p=L_{0} p+\frac{1}{\sqrt{\varepsilon}} L_{1} p+\frac{1}{\varepsilon} L_{2} p=0
$$

and expanding

$$
p=p_{0}+\sqrt{\varepsilon} p_{1}+\varepsilon p_{2}+\cdots
$$


gives to order $1 / \varepsilon$

$$
L_{2} p_{0}=\frac{\partial p_{0}}{\partial \tau}+K p_{0}=0
$$

It follows from the ergodocity of $\xi_{1}$ that the only bounded solution of (A.4) is constant with respect to $\tau$ and $\xi_{1}$. Thus

$$
p_{0}=p_{0}(y, t) \text {. }
$$

At order $1 / \sqrt{\varepsilon}$ we obtain

$$
L_{2} p_{1}=-L_{1} p_{0}=-\sum_{i=1}^{n} Y_{1}^{i}\left(y, \tau, \xi_{1}\right) \frac{\partial p_{0}}{\partial y_{i}} .
$$

By assumption (ii) of Theorem 2.1, the right-hand side of (A.6) satisfies the solvability condition (Fredholm alternative) for the operator $L_{2}$; i.e., the right-hand side is orthogonal to the solution $p^{*}$ of the adjoint equation

$$
L_{2}^{*} p^{*}=0 .
$$

The solution is given by

$$
\begin{aligned}
p_{1} & =-L_{2}^{-1} \sum_{i=1}^{n} Y_{1}^{i}\left(y, \tau, \xi_{1}\right) \frac{\partial p_{0}}{\partial y_{i}} \\
& =\int_{0}^{\infty} d s \int_{\mathbb{R}^{m_{1}}}\left[P\left(s, \xi_{1}, d z\right)-\mu(d z)\right] \sum_{i=1}^{n} Y_{1}^{i}(y, \tau+s, z) \frac{\partial p_{0}}{\partial y_{i}} \\
& =Q_{p_{0}}\left(y, \tau, \xi_{1}\right) .
\end{aligned}
$$

At order 1 we obtain

$$
L_{2} p_{2}=-L_{0} p_{0}-L_{1} p_{1} .
$$

Again, the solvability condition for (A.9) is that the right-hand side of (A.9) has to be orthogonal to $p^{*}$ given by (A.7). Thus after substituting $p_{1}$ from (A.8) into the right-hand side of (A.9) the solvability condition gives

$$
\begin{aligned}
\frac{\partial p_{0}}{\partial t}+ & \lim _{T \rightarrow \infty} \frac{1}{T} \int_{t_{0}}^{t_{0}+T} \int_{\mathbb{R}^{m_{1}}} \mu\left(d \xi_{1}\right) \\
& \times \sum_{i=1}^{n}\left[Y_{1}^{i}\left(y, \tau, \xi_{1}\right) \frac{\partial Q_{p_{0}}\left(y, \tau, \xi_{1}\right)}{\partial y_{i}}+Y_{0}^{i}(y, \tau) \frac{\partial p_{0}}{\partial y_{i}}\right] d \tau=0 .
\end{aligned}
$$

It can be shown, by some lengthy algebra (that we omit here), that Eq. (A.10) is equivalent to $\left(\partial p_{0} / \partial t\right)+\bar{L} p_{0}=0$, where $\bar{L}$ is given by (2.6), (2.7). 
Finally, it follows from the ergodicity of $h(\tau, \cdot)$ and the averaging with respect to $\tau$ in (A.10) that $p_{0}(y, t)$ is independent of $\mathbf{H}_{\tau}$ and whence (A.10) is the backward Kolmogorov equation for the diffusion process $\bar{y}(t)$ defined by the Itô equation (2.8).

Next we prove Proposition 2.2.

Proof of Proposition 2.2. The proof follows from Theorems 2.1 and 2.2 and the following observation.

By assumption $X_{2}\left(x, \xi_{2}\right)$ is a smooth function of $x$ and, thus, so is $h(\tau, y)$. Therefore,

$$
h\left(\tau, y_{2}\right)=h\left(\tau, y_{1}\right)+\frac{\partial h}{\partial y}\left(\tau, y_{1}\right)\left(y_{2}-y_{1}\right)+R\left(y_{2}-y_{1}\right) .
$$

By assymption $|\partial h(\tau, y) / \partial y| \leqslant C$ and higher order derivatives of $h(\tau, y)$ are uniformly bounded by powers of $|y|$. This gives

$$
\left|h\left(\tau, y_{2}\right)-h\left(\tau, y_{1}\right)\right| \leqslant C\left|y_{2}-y_{1}\right|+r\left(\left|y_{2}-y_{1}\right|\right),
$$

where $r\left(\left|y_{2}-y_{1}\right|\right) /\left|y_{2}-y_{1}\right| \rightarrow 0$ as $y_{2} \rightarrow y_{1}$ uniformly in $\tau$. Finally, (A.12) gives

$$
|x(t)-\bar{x}(t)| \leqslant C|y(t)-\bar{y}(t)|+r(|y(t)-\bar{y}(t)|)
$$

and the statements of the proposition follow.

\section{REFERENCES}

1. E. WONG AND M. ZAKAI, On the relationship between ordinary and stochastic differential equations, Internat. J. Engrg. Sci. (1965).

2. G. C. Papanicalaou and W. Kohler, Asymptotic theory of mixing stochastic differential equations, Comm. Pure Appl. Math. 27 (1974).

3. G. Blankenship and G. C. Papanicalaou, Stability and control of stochastic systems with wide-band noise disturbances, I, SIAM J. Appl. Math. 34 (1978).

4. R. Bellman, J. Bentsman, and S. M. Meerkov, Nonlinear systems with fast parametric oscillations, J. Math. Anal. Appl. 97 (1983).

5. R. Bellman, J. Bentsman, and S. M. MeErkov, Vibrational control of nonlinear systems: Vibrational stabilizability, IEEE Trans. Automat. Control AC-31 (1986).

6. L. Arnold, H. Crauel, and V. Winstutz, Stabilization of linear systems by noise, SIAM J. Control Optim. 21, No. 3 (1983), 451-461.

7. M. F. NoRman, Ergodicity of diffusion and temporal uniformity of diffusion approximation, J. Appl. Probab. 14 (1977).

8. L. ARnold, G. C. Papanicalaou, and V. Wihstutz, Asymptotic analysis of the Lyapunov exponent and rotation numbers of the random harmonic oscillator and applications, SIAM J. Appl. Math. (1986).

9. L. ARNOLD, "Stochastic Differential Equations: Theory and Applications," Wiley, New York, 1974. 